\title{
Sitzung vom 10. November 1879 .
}

Vorsitzender: Hr. A. W. Hofmann, Präsident.

Das Protocoll der letzten Sitzung wird genehmigt.

Hr. Tiemann verliest darauf das unten abgedruckte Protocoll der Vorstands-Sitzung rom 2. November.

Zu ausserordentlichen Mitgliedern werden proclamirt die Herren:

Carl Böhringer, Chemiker in Bönnigbeim [Württemberg];

W yndham Rowland Dunstan, London;

O. Kraft, Fabrikdirector, St. Petersburg;

Charles Violette, Professor der Chemie in Lille;

Carlos Serzedello, in Lissabon;

Ernst Krause, in Saarbrücken;

$\left.\begin{array}{l}\text { Alois Sroboda, } \\ \text { Wenzel Kolár, }\end{array}\right\}$ am k. k. Polytechnicum in Prag;

Dr. Hake, in Hampshire (England);

Jules Naville, Fos par Istres, Bouches du Rhône;

William Conrad, in Würzburg;

Eg. Wild, in Zürich;

M. Maisch, Professor in Philadelphia;

H. v. Knapp, in Berlin, Chem. Univ.-Laborat.

Zu ausserordentlichen Mitgliedern werden vorgeschlagen die Herren :

John J. Thomsen, Harward College, Cambridge U. S. A. 34, Weld Hall (durch A. W. H ofmann u. A. L. Thomsen); Ed. Keller, Zürich, Polytechnic. (durch C. Hell und $O$. Stüber);

Dr. Carl Brunnemann, Assistent am chem. Universităts-

Laboratorium Greifswald (durch H. Limpricht and H. Schwanert);

Hermann Schmalzigang, Mailand, Fabricca Lombarda di Prodotti Chimici (durch W. Körner und C. Forst); 
W. Harmsen, Rostock, Chem. Universit.-Laborat. (durcb O. Jacobsen und C. Brunnengräber);

Agostino Oglialoro, Professor der Chemie an der Universität Messina (durch S. Canizzaro und R. Scbiff);

Dr. G. Carnelut ti, Vice-Director am Istituto Cbimico, Panisperna Rom (durch dieselben);

Dr. Paul Jeserich, Berlin, Klosterstr. 49 (durch Engen Sell und B. Proskauer);

James Eisenberg, Dorotheenstr. 68 (durch S. Gabriel und F. Krüger).

Für die Bibliothek sind als Geschenk eingegangen:

Chemiker-Kalender für das Jahr 1880. Herausgegeben von Rud. Biedermann. Erster Jahrgang. I. Theil. Berlin 1880.

Tommasi, Donato. Ricerene sulle formole di costituzione dei composti ferrici. Parte prima. Idrati ferrici. Sep-Abdr. Firenze 1879.

Der Schriftführer: Der Vorsitzende:
A. Pinner.
A. W. H of mann.

Protocoll der Vorstands-Sitzung vom 2. November 1879.

Anwesend die Herren: A.W. Hofmann, A. Bannow, E. Baumann, A.Franck, S.Gabriel, A.Geyger, G.Krämer, C.Liebermann, A. Pinner, E. Salkowski, C. Sarnow, C. Scheibler, E. Schering, Eug. Sell, F. Tiemann, H. Wichelbaus.

1) Es wird beschlossen, die ordentliche General -Versammlung auf Freitag, den 19. December anzuberaumen; dieselbe soll im grossen Hörsaale des chemischen Universitäts-Laboratoriums Georgenstrasse No. 35 stattfinden.

2) Der Scbriftüurer zeigt an, dass in Gemässheit von $\S 3$ der Statuten rechtzeitig Vorscbläge zur Wahl von zwei Ebren-Mitgliedern gemacht wordeu sind.

3) Der Schriftführer berichtet über den Druck des GeneralRegisters über die ersten zehn Jahrgänge der Berichte und theilt mit, dass bis zum 1. November etwa 700 Bestellungen auf dieses Werk ron Mitgliedern der Gesellschaft beim Secretariat eingelaufen sind.

Der Vorstand beschliesst, eine erneute Aufforderung zum Abonnement auf das General-Register auf dem Umschlage des nächsten Heftes der Berichte abdrucken zu lassen.

Das Secretariat wird beauftragt, in einem Schreiben 1) Hrn. Dr. C. Bisch off zu ersuchen, die durch Satzänderungen etc. veranlassten aussergewöhnlichen Correcturen beim Druck des GeneralRegisters nach Möglichkeit einzuschränken, und 2) denselben zu ermäcbtigeu, die fertig gestellten Druckbogen mit dem Imprimatur zu verseben. 
4) Das Bürean wird beauftragt, an Hrn. Gebeimen Bergrath Haucbecorne ein Dankschreiben für die bereitwillige Ueberlassung des bisherigen Sitzungs-Saales der Gesellschaft zu richten.

5) Der Vorstand kommt auf den unter No. 2 des letzten SitzungsProtocolles verzeichneten, die Erhöhung der Mitglieder-Beiträge betreffenden Antrag zurück. Der Vorstand hatte das Secretariat beauftragt, die Ansicbten der auswärtigen Vorstands-Mitglieder über diesen Antrag einzuholen. Der Schriftuährer verliest die Antwortschreiben, welche auf das rom Secretariat erlassene Rundschreiben eingetroffen sind. Sechs der auswärtigen Vorstands-Mitglieder, nämlich die HHrn. Peter Griess, F. Hoppe-Seyler, A. Laubenbeimer, A. Lieben, R. Ot to and $O$. Wallach erklären sich mit dem Antrage des Vorstandes vollständig einverstanden. Hr. Lothar Mey er glaubt zu dem Antrage eine besondere Stellung nicht nebmen zu sollen, ${ }_{n}$ weil keine der für die Entscheidung wichtigen Einzelheiten zu seiner Kenntniss gekommen ist". Herr R. Fittig bätte eine gleichmässige Erhöhung der Beiträge aller Mitglieder gewünscht. Auch Hr. R. Hof fmann beanstandet, dass von der beabsichtigten Erböhung der Beiträge nur die answärtigen Mitglieder der Gesellschaft betroffen werden und fragt an, ob dem wachsenden finanziellen Bedürfniss nicht besser darch eine Reduction der in die Berichte aufzunehmenden $\mathbf{A b}$ bandlungen entgegen zu arbeiten sei? Aus den in den Schreiben der HHrn. Fittig und Hoffmann enthaltenen Gründen und besonders um die Aufnabme von mittelmässigen und unbedeutenden Abhandlungen zu erschweren, welche, $n$ anknüpfend an unvollständig beobachtete Thatsacben mit historischen Excursen beginnen und mit theoretischen Speculationen über die Constitution oberflächlich studirter oder überhaupt noch nicht dargestellter Verbindungen endigen", mithin mehr Raum beanspruchen, als sie verdienen, spricht Hr. W. Lossen sich gegen eine Erböhung der Mitglieder-Beiträge aus.

Der Vorstand bescbliesst, den Inhalt der genannten Schreiben ausfübrlich zur Kenntniss der General-Versammalung zu bringen und fordert den Redacteur und die Publications-Commission ron Neuem auf, bei der Aufnahme von Abbandlungen in die Berichte möglichst streng zu verfahren. Die Beamten werdeu gleichzeitig beauftragt, genaue Erhebungen über die Ausgaben anzustellen, welche durch die besonders deu Berliner Mitgliedern za gut kommenden Einrichtungen: Bibliothek, Sitzungs-Saal etc. veranlasst werden. Diese Erbebungen sollen der General-Versammlung unterbreitet werden.

6) Die erste Sitzung der Gesellschaft im Jabre 1880 soll am Montag, den 12. Januar stattfinden.

Der Schriftführer:

Ferd. Tiemann.
Der Vorsitzende:

A. W. Hofmann. 